\title{
Interaction Design of Smart Home Mobile Control Terminal
}

\author{
Jieping $\mathrm{Hu}^{1}$, Nan Yang ${ }^{2}$, Shulin Yang ${ }^{3}$ and Bin Wang ${ }^{3}$ \\ ${ }^{1}$ Department of Basic, Beijing Institute of Graphics Communication, Beijing, China \\ ${ }^{2}$ General Education College, Beijing International Studies University \\ ${ }^{3}$ College of Information Engineering, Beijing Institute of Graphics Communication, Beijing, China
}

\begin{abstract}
With the wide application of mobile terminal in smart home system, the interaction design of mobile terminal becomes more and more important. Interaction design is different from a simple interface design, it will combine interface view, process logic, interactive manner, taking into account the beautiful interface, software implementation and user experience. The design scheme presented in this project embodies the design concept of simplicity, beauty, convenience, adaptability and customization. The application in the practical system shows that this scheme enhances the user experience, has the characteristics of easy to implement, easy to expand and installation.
\end{abstract}

Keywords-interaction design; smart home; design of device com ponents

\section{INTRODUCTION}

Smart home can provide us with healthy, convenient and comfortable life, so people are choosing a lifestyle smart home, smart home terminal should be presented to the user a high degree of reliability and good looking, easy to use interface, allow people to fully experience the comfort and convenience of smart home life. Smart mobile devices as smart home control terminal in addition to give users a convenient and good man-machine interface. Users exchange information with mobile terminals through human computer interaction. Therefore, the interactive experience directly related to the performance of the system can give full play to the user can accurately, efficiently, easily and happily, the system is very important for [1]. Interaction design refers to the software human-computer interaction, operating logic, interface structure, software implementation design. Good interaction design is not only let the software becomes individual taste, but also make the operation of the software becomes comfortable, simple and free, fully embodies the orientation and characteristics of software[2], and allows users to customize the software interface, convenient operation and easy control, software realization. In this paper, we will study the interactive design of mobile terminal based on Android system.

\section{DESIGN PRINCIPLE AND StRUCTURE}

\section{A. Design Principle}

Smart home mobile control terminal need to offer convenient, visual operating interface, allows the user to control different devices in the room. You need to follow a few principles:
1) Simplicity: interface structure is simple, the operation is simple, convenient for users.

2) Beautiful: elegant interface, reasonable layout, color coordination, visual effect is good.

3) Convenience: can easily select home floor, room and equipment; Intuitive way to present family smart devices, and convenient for operation and control of family intelligent devices.

4) Adaptability, can according to different family intelligent device and the number of different, automatically increase or decrease interface components.

5) Customization: users can according to their own need to create patterns, such as home, leave home mode, sleep patterns. Users can also set up a common scene model based on preferences.

\section{B. Interface Integral Structure}

After the user login successfully enter the main interface, as shown in Figure I. The main interface can display the outdoor weather, temperature, wind, humidity and indoor temperature, the current date and time; also can choose the status, home furnishing, security, service, scenes, settings and other functions into different sub function interface. Select home to enter the home control interface, in the home control interface, you can press the floor, room, equipment set, the level of equipment to open the device interface as shown in Figure II.

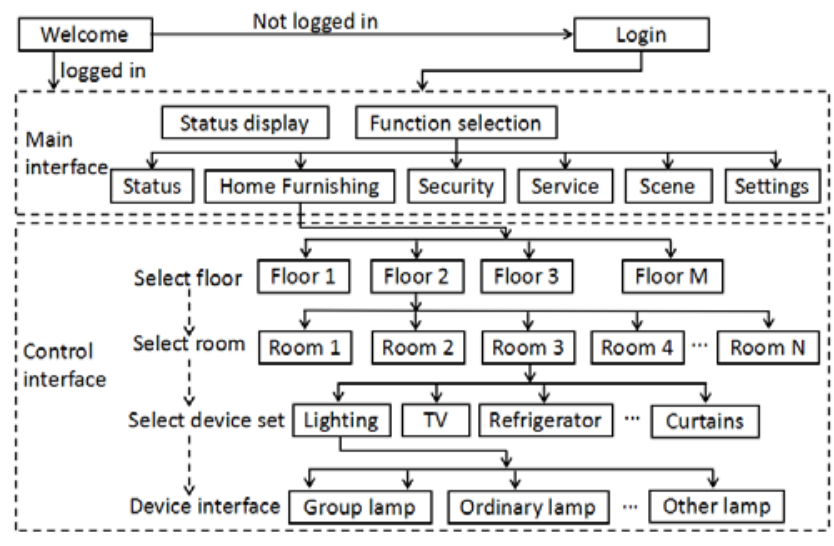

FIGURE I. INTERFACE INTEGRAL STRUCTURE 


\section{Main Interface}

After successful login, enter the system main interface, as shown in Figure II. Main interface display outdoor weather, temperature and wind conditions, indoor temperature, humidity, and the current date and time. In the main interface can enter six sub-functions. The main interface uses FrameLayout layout. The view added to this layout is displayed in a cascading manner. In this layout to add two LineLayout layout, the first linear layout to place the weather, calendar, placed the second linear layout function button.

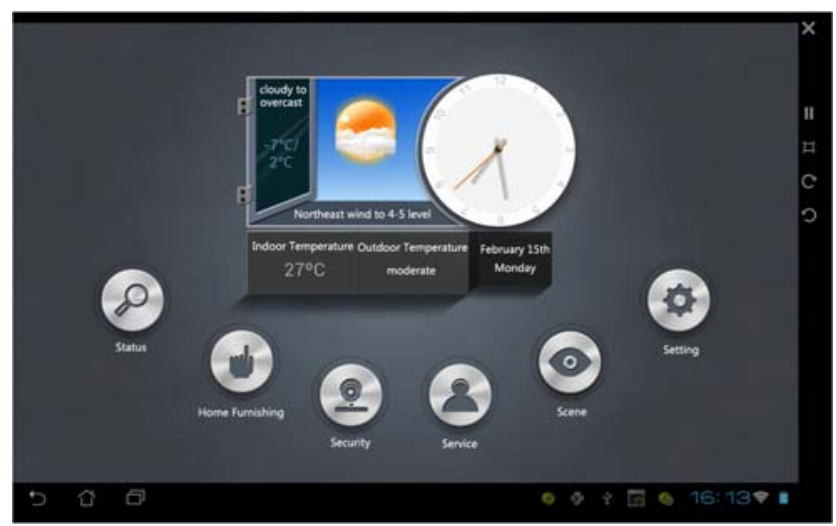

FIGURE II. MAIN INTERFACE

At the main interface, click "home furnishing" function button, enter the home control interface, as shown in Figure III. At the interface, the left is a list of floors, and the right side of this list is a list of rooms. Select the device set in the middle to enter the device set. Scene list on the right. Select the scene to control the device by device settings.

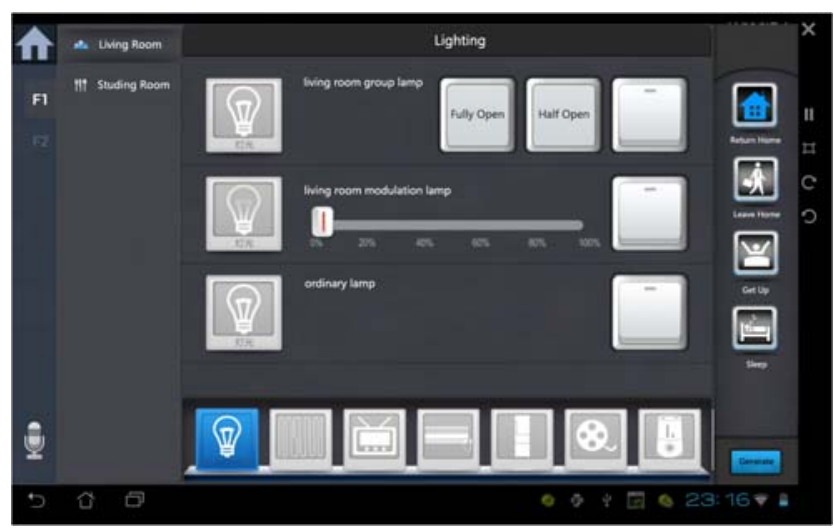

FIGURE III. CONTROL INTERFACE

\section{DESIGN OF DEVICE COMPONENTS}

\section{A. Design Principles of Device Components}

Android provides sophisticated and powerful components of the model to build the user's UI. Mainly based on the layout class: View and ViewGroup. On this basis, the Android platform provides a large number of prefabricated View and ViewGroup subclass, layout and widgets. You can use them to build their own UI. If the prefabricated widgets do not meet your requirements, you can create your own view. If only making small adjustments for existing widget or layout, inherit the class, covering the relevant methods, which define the user component. User component can more accurately control the appearance and function of view elements.

Smart home mobile control terminal interface components, mainly adopts created in three ways:

1) Adjusting components: inherited subclass of View, expand the existing Android components.

2) Complete custom components: inherited View class, interface and event completely controlled by yourself.

3) Synthetic components: inheritance ViewGroup or its subclass, combining existing Android components.

Smart home mobile control terminal, device component is the most commonly used variety of function buttons, such as button switches, mode button, command button, increasing or reducing button. a different button message is different, showing different ways.The way of adjustment is adopted to design function button. Function buttons extend TextView.

Other device components are mainly used in the way of the synthesis of components, that is not completely customized to a new view of the component, but the existing view components combined together to deal with common business logic. The inheritance of ViewGroup or its sub class Layout layout, other layout can also be nested in them, like Activity, you can use the container component to create the XML statement based on, can also be embedded in the program code. Similar to the existing Android components, if the custom components can be designed in XML, can achieve the separation of interface design and code design, provides greater flexibility and convenience for maintenance interface, it can avoid the cumbersome code. However, due to the smart home terminal interface according to the different families of the device dynamic assembly, the use of Java code control interface, can be more controlled, but the development process is cumbersome, not easy to update and maintenance. If you can do it, you can use the XML file configuration device components, and through the Java code to create the device component object, which is the most rational. It is mainly used in the construction method of the device components to use the layout file similar to the following statement, and get the view.

View view $=$ ((Activity) getContext()). getLayoutInflater(). inflate(R. layout.groupLamp, this).

\section{B. Design of Device Components Class}

Using object-oriented mechanism design equipment components, first define a device base class, packaging equipment data, equipment state data and instructions controller. The class inheritance LinerLayout and implements the Ysl_ClickListener interface. In addition, refreshUI() is an abstract method, used to update the interface, and be realized by the subclass. Component hierarchy as shown in Figure IV. 


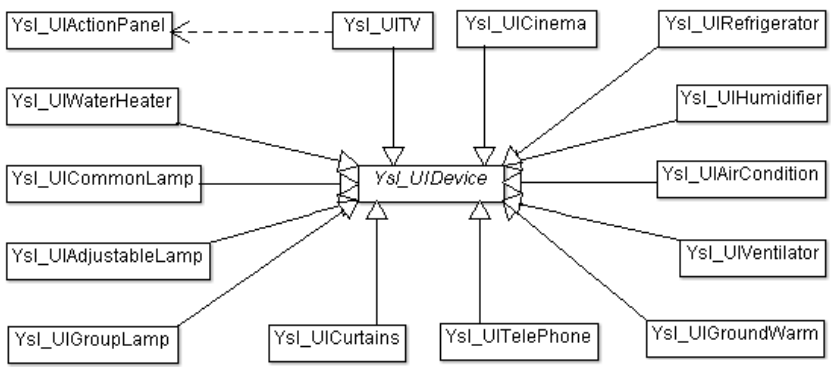

FIGURE IV. STRUCTURE OF DEVICE COMPONENTS CLASS

\section{COMMUNiCATION MOdULE INTERACTION WITH THE DEVICE COMPONENTS}

\section{A. Device Components Send Instructions}

In order to facilitate the dynamic update or increase device components, the device components are built in a separate proj ect. A network communication module for transmitting instruc tions to device components is in the main project, and the devi ce components is in a device project, belong to a different pac kage. The Ysl_ComCommand instance is passed to the device component object when a device component object is created. The device components sends instructions to achieve mainly $t$ hrough the function buttons. When the function button is click ed, the onClick event is called, and the following method is cal led in the event to realize the instruction sending.

public void sendInstruction(String instructionName,

String instructionValue) \{

try \{

Method method = command. getClass(). getMethod( "sendDa ta", new Class[] \{ Context. class, Handler. class, String. class, Stri ng. class, String. class, String. class \});

method. invoke(command, this. getContext(), handler, device Data. get("deviceId"). toString(), instructionName, instructionValu e);

$$
\begin{array}{r}
\text { \} catch (Exception e) }\{ \\
\text { e. printStackTrace(); }
\end{array}
$$$$
\}
$$

The above method uses the Java reflection mechanism to c all the sendData () method of Ysl_ComCommand instance[5].

\section{B. Communication Thread Refreshing Device Component Interface}

In the Android design ideas, in order to ensure the user to smooth operation experience. Some time-consuming task can not be run in the UI thread, like access to the network was part of this task. So we have to re-open a sub-thread to run these tasks, such as accessing the network. So we have to re open a child thread to run these tasks. However, often these tasks will eventually be directly or indirectly required to access and control UI controls. For example, access to the network to obtain data, and then need to be processed to display these data. But Android rule that other threads can not access those UI controls in addition to the UI thread. Because, when the thread is related to the operation of UI operation, will cause danger to the main thread, that is to say, can only be updated in the main thread, it is dangerous to operation in the child thread.

For this, handler is used to solve this problem. Handler is a tool used to transfer information between threads, Handler mainly receives the data sent by the thread, and uses this data to update UI, which is used to interact with the UI main thread. In order to avoid the device interface components associated with the Handler instance can not be recycled in a timely to generate a memory leak, static internal class is used. The static internal class does not hold a reference to the external class, so it does not result in a memory leak outside class instance.

\section{CONCLUSIONS}

The interaction design of mobile control terminal is very important in the design of smart home system. The interface view, process logic, combined interaction design, can better increase the user experience. The design scheme presented in this paper embodies the design concept of simplicity, beauty, convenience, adaptability and customization, which is easy to implement, easy to install and easy to install.

\section{ACKNOWLEDGMENT}

This paper was supported by National Natural Science Foundation "Analysis and Solution of Nonlinear Dynamic Process in High Speed Printing Equipment”(No.61272030) and "onlinear Dynamics model and Analysis of Gear Systems for Offset Press” (No. 61472461).

\section{REFERENCES}

[1] Zhang Qingfeng /translation, The human-computer interaction (third edit ion), Beijing: electronic industry press, 2007.

[2] Liu Xingwang. UI design analysis. http://blog.renren.com/share/2258425 04/5337149904.

[3] Yu Yanan. Research and implementation of human computer interaction based on Android platform in China (2011).http://www.paper.edu.cn.

[4] Zhao Liang, ZHang Wei, “ Research and Design of Interface Based on A ndroid Technology”, Computer Knowledge and Technology,2009, Vol.5, 29, pp.8183-8185

[5] How to implement custom components of Android custom components. http://blog.csdn.net/jjwwmlp456/article/details/41076699.

[6] Principle of Java reflection mechanism and its application in Android. ht tp://407827531.iteye.com/blog/1308702.

[7] Comparison of advantages and disadvantages of AsyncTask and Handler. http://blog.csdn.net/onlyonecoder/article/details/8484200. 